\title{
AUDIT
}

\section{Safety of the insulin tolerance test}

\section{P J Galloway, E McNeill, W F Paterson, M D C Donaldson}

Concerns have been raised about the hazards of the insulin tolerance test (ITT), used to measure growth hormone secretion. In Glasgow, we continue to use this test, adhering to a strict protocol. A review of outcome over a 10 year period (1989-99), during which 550 ITTs were performed, was undertaken. No serious adverse events occurred; in particular, no child fitted or required intravenous glucose. Fewer tests were done during the latter five years, with a higher yield of growth hormone $(G H)$ deficiency, reflecting our increasingly conservative approach to paediatric $\mathrm{GH}$ therapy during this period. We conclude that the ITT is safe and reliable in a paediatric setting provided that a strict procedure is followed.

See end of article for authors' affiliations

.....................

Dr P Galloway, Consultant Clinical Biochemist, Department of Biochemistry, Royal Hospital for Sick Children, Yorkhill, Glasgow G3 8Sj, UK; petergalloway@ lineone.net

Accepted 28 June 2002
1 n 1991, following several fatalities in the UK, the Department of Health issued a circular ${ }^{2}$ warning of the hazards of the insulin tolerance test (ITT), stating that: "the insulin tolerance test should not be used in children when only growth hormone reserve needs to be tested".

Since then many paediatric centres have abandoned the ITT as a means of assessing growth hormone $(\mathrm{GH})$ reserve although it remains the standard diagnostic test for GH deficiency in adults, in view of its sensitivity and reproducibility. ${ }^{3}$ We have continued to use the ITT as our first line test, reserving the arginine test for children with epilepsy or cardiac disease and those under 5 years.

We have reviewed retrospectively our experience of performing ITTs over a 10 year period (1989-99), with particular attention paid to the last five years, during which a dedicated nurse specialist has been overseeing the tests. Our principal aim was to assess the safety of the ITT, defining the key morbidity measures as: (a) hypoglycaemia requiring emergency administration of intravenous glucose; (b) hypoglycaemic convulsions; and (c) death. We have also examined the timing of the glucose nadir following insulin administration as this has practical implications for oral glucose administration. Finally, in the light of a change in the pattern of $\mathrm{GH}$ prescribing in Scotland over the past 10 years, ${ }^{5}$ we have noted the annual number of ITTs performed.

\section{METHODS}

The following strict ITT protocol was adhered to:

- Child must be fasted overnight.

- Blood glucose must be $>3.0 \mathrm{mmol} / \mathrm{l}$ at time -30 minutes and 0 minutes.
- Oxygen, glucose, and hydrocortisone must be available.

- A doctor must be present for 45 minutes following insulin administration and a nurse specialist throughout. (Protocol since 1995, when an endocrine nurse specialist was appointed. During the first five years of the study period, endocrine tests were carried out on a paediatric inpatient ward by junior medical staff.)

- Child must eat and remain on ward for one hour before cannula removal and discharge home.

- Administration of insulin. An insulin concentration of 1 unit $/ \mathrm{ml}$ is used and a dose of 0.15 units $/ \mathrm{kg}$ is given (0.1 units $/ \mathrm{kg}$ if panhypopituitarism is suspected). The doctor must sign for the insulin and the nurse must check the dosage.

- Blood sampling. Glucose and growth hormone concentrations are measured in $4 \mathrm{ml}$ samples drawn at $-30,0,15,30,60,90$, and 120 minutes. The rationale of the -30 minute sample is to capture any stress related GH surge, as this may be followed by a refractory period, giving the misleading interpretation of GH deficiency.

- Management of hypoglycaemia. If the blood glucose drops below $2.2 \mathrm{mmol} / \mathrm{l}$ and/or the child is symptomatic, $30-40 \mathrm{ml}$ of Lucozade (up to $10 \mathrm{ml} / \mathrm{kg}$ body weight) is given. If the child becomes unrousable or is fitting, $0.5 \mathrm{~g} / \mathrm{kg}$ dextrose is given intravenously, and blood glucose rechecked. Thereafter 50-100 mg hydrocortisone, followed by a $10 \%$ dextrose infusion at $0.1 \mathrm{ml} / \mathrm{kg} / \mathrm{min}$ is given. A further dextrose bolus is administered only if blood glucose remains $<6.0 \mathrm{mmol} / \mathrm{l}$.

\section{Statistical analysis}

The relative risk of a future adverse event occurring as a result of the procedure, assuming that none had occurred to date, was calculated according to a simple formula, first described by Hanley in $1983,{ }^{67}$ which states that:

the upper limit of the $95 \%$ confidence inter$\mathrm{val}$ of the probability of such an event (i.e. maximum risk) $=3 / n$ (for $n>30$ )

where $\mathrm{n}=$ number in series (i.e. number of past, uneventful procedures).

Abbreviations: $\mathrm{GH}$, growth hormone; ITT, insulin tolerance test 


\section{RESULTS}

A total of 550 ITTs were performed between 1989 and 1999. No serious adverse events occurred. In particular, no child lost consciousness, fitted, or required intravenous glucose following hypoglycaemia. The upper limit of the confidence interval for relative risk from the procedure is therefore $3 / 550=0.005$ $=0.5 \%$. Glucose profiles were documented for the 223 ITTs performed since 1995. The glucose nadir occurred at 15 minutes in $118(53 \%)$ cases (range $0.6-2.2 \mathrm{mmol} / \mathrm{l}$ ) and at 30 minutes in $105(47 \%)$ cases (range $1.1-2.3 \mathrm{mmol} / \mathrm{l}$ ). Between 1989 and 1992 the annual number of ITTs performed ranged from 57 to 77. During this period GH values of $10-20 \mathrm{mU} / \mathrm{l}$ were recorded in 27\%. From 1993 to 1999, the annual number of ITTs performed decreased to 37-52 and the number of cases with $\mathrm{GH}$ values of $10-20 \mathrm{mU} / \mathrm{l}$ rose to $43 \%$. Over the 10 year period $\mathrm{GH}$ values $<10 \mathrm{mU} / \mathrm{l}$ were recorded in $14 \%$. GH concentrations were $>20 \mathrm{mU} / \mathrm{l}$ at time -30 minutes in $16 \%$, indicating a normal $\mathrm{GH}$ response to stress, thus precluding $\mathrm{GH}$ deficiency.

\section{DISCUSSION}

In this assessment of the safety of the ITT in childhood we report no serious adverse events in the 550 cases studied. We acknowledge that this finding cannot preclude adverse events occurring in future patients but the probability, based on the numbers of patients studied, is, at most, 5 per thousand. Indeed no test that alters glucose homoeostasis can be regarded as safe. Even the simple act of fasting a severely hypopituitary child may cause symptomatic hypoglycaemia. However, our experience indicates that the ITT is relatively safe provided that this endocrine investigation is carried out in a well resourced, specialised setting by experienced, competent staff. A similar conclusion was reached by Jones et al, who carried out an audit of 161 ITTs performed in adults during a 12 month period. ${ }^{8}$ In this study one serious adverse event (a grand mal fit) occurred, but the patient recovered rapidly with no neurological sequelae following administration of intravenous dextrose and hydrocortisone.

It was not possible, in a retrospective study of this nature, to evaluate the degree of distress which may have been caused by the process of venepuncture, and by symptoms of insulin induced hypoglycaemia such as drowsiness, sweating, and malaise. Nor was it our remit to compare the symptoms of insulin induced hypoglycaemia with those caused by other pharmacological tests of GH secretion, such as clonidine (for example, drowsiness, hypotension), ${ }^{9-11}$ or glucagon (for example, nausea, headache, sweating, vomiting). ${ }^{12}$

During the 10 year study period an endocrine specialist nurse was appointed (1995) and a ward dedicated for day case investigation of children was established (1997). While it would be difficult to measure objectively an improvement in service resulting from these changes, we have no doubt that the standard of day care is much improved. Moreover, in the light of the recent changes to the working practice of junior medical staff, we consider that our endocrine day case service would be untenable without the consistent input from a specialist nurse.

The glucose nadir occurred at 15 minutes in just over half of our patients and by 30 minutes in virtually all. This finding has led us to favour the pre-emptive use of Lucozade (a readily available $20 \%$ glucose drink) at 15-20 minutes to avoid more profound hypoglycaemia developing.

Since 1993, we have been more selective in our approach to growth hormone therapy. ${ }^{5}$ This is reflected in a decrease in the number of children tested and a higher yield of subjects with peak $\mathrm{GH}$ values of $<20 \mathrm{mU} / \mathrm{l}$, the biochemical measure of $\mathrm{GH}$ deficiency, and indicates improved targeting of children likely to benefit from investigation.

Not only are we satisfied as to the relative safety of the ITT when performed by experienced staff using a strict protocol, but we would also argue that it is in many ways preferable to other tests of GH reserve. The stimulus of hypoglycaemia, a naturally occurring phenomenon, is arguably more physiological than that caused by pharmacological agents. Moreover, insulin is easily administered and the resultant hypoglycaemia is readily reversible. The elective use of Lucozade ameliorates the symptoms of hypoglycaemia without affecting the counter regulatory response.

Other methods of GH stimulation are not without their drawbacks. While arginine administration does not result in hypoglycaemia - the increase in insulin release is balanced by a concomitant increase in glucagon, resulting in a net rise in plasma glucose ${ }^{13}$-we have encountered problems with venous occlusion caused by the viscosity of the infusion, sometimes necessitating replacement of the intravenous cannula. We have no experience of other agents, but it is well recognised that clonidine causes unpleasant symptoms such as drowsiness and hypotension which are not rapidly reversible. ${ }^{9}{ }^{10}$ In addition, clonidine has recently been shown to be associated with hypoglycaemia, although the mechanism of action is unclear. ${ }^{11}$ Glucagon administration causes nausea and vomiting, ${ }^{12}$ and may also result in hypoglycaemia by stimulating insulin release. ${ }^{14}$

Our observation that no child required intravenous dextrose in the treatment of hypoglycaemia emphasises that in virtually all cases oral glucose administration is all that is required. Indeed, fatalities associated with the ITT have involved the administration of large quantities of intravenous dextrose. There is no place for large quantities of either hypotonic (for example, $5 \%$ ) or hypertonic (50\%) dextrose in insulin induced hypoglycaemia. If necessary, the child should merely receive $0.5 \mathrm{~g} / \mathrm{kg}$ of dextrose as a $10 \%$ solution, followed by $10 \%$ dextrose with $0.45 \%$ saline at maintenance rates.

In highlighting the favourable safety record of the ITT in our hospital we are not advocating the widespread reintroduction of this method of anterior pituitary function testing, especially in departments where relatively small numbers of children are involved. Indeed, given the controversies surrounding the selection of children for investigation and treatment of $\mathrm{GH}$ deficiency, ${ }^{4}{ }^{15}$ we believe that the decision to embark on growth hormone stimulation testing should only be made by paediatricians who are experienced/trained in paediatric endocrinology, and/or working in close collaboration with a regional centre.

\section{Authors' affiliations}

P J Galloway, Department of Biochemistry, Royal Hospital for Sick Children, Yorkhill, Glasgow, UK

E McNeill, W F Paterson, M D C Donaldson, Department of Child Health, Royal Hospital for Sick Children

\section{REFERENCES}

1 Shah A, Stanhope R, Matthew D. Hazards of pharmacological tests of growth hormone secretion in childhood. BM 1992;304:173-4.

2 DOH. Anterior pituitary function tests in childhood. London: Department of Health, 1992.

3 Hoffman DM, O'Sullivan AJ, Baxter RC, et al. Diagnosis of growth-hormone deficiency in adults. Lancet 1994;343:1064-8.

4 Saggese G, Ranke MB, Saenger $P$, et al. Diagnosis and treatment of growth hormone deficiency in children and adolescents: towards a consensus. Horm Res 1998;50:320-40.

5 Paterson WF, Donaldson MDC, Greene SA, et al. The boom that never was: results of a 10 year audit of paediatric growth hormone prescribing in Scotland. Health Bull 2000;60:457-66.

6 Hanley JA, Lippman-Hand A. If nothing goes wrong, is everything all right? Interpreting zero numerators. JAMA 1983;249:1743-5.

7 Eypasch E, Lefering R, Kum CK, et al. Probability of adverse events that have not yet occurred: a statistical reminder. BM 1995;31 1:619-20.

8 Jones SL, Trainer PJ, Perry L, et al. An audit of the insulin tolerance test in adult subjects in an acute investigation unit over one year. Clin Endocrinol 1994;41:123-8.

9 Gil-Ad I, Topper E, Laron Z. Oral clonidine as a growth hormone stimulation test. Lancet 1979;2:278-9. 
10 Lanes R, Recker B, Fort P, Lifshitz F. Low-dose oral clonidine. A simple and reliable growth hormone screening test for children. Am J Dis Child $1985 ; 139: 87-8$

11 Huang C, Banerjee K, Sochett E, et al. Hypoglycaemia associated with clonidine testing for growth hormone deficiency. J Pediatr 2001;139:323-4.

12 Leong KS, Walker AB, Martin I, Wile D, et al. An audit of 500 subcutaneous glucagon stimulation tests to assess growth hormone and
ACTH secretion in patients with hypothalamic-pituitary disease. Clin Endocrinol 2001:54:463-8.

13 Massi-Benedetti F, Sposito M, Barboni G, et al. Blood glucose, plasma insulin and glucagon response to arginine in infants during the first month insulin and glucagon response to arginine in

14 Hindmarsh PC, Swift PGF. An assessment of growth hormone provocation tests. Arch Dis Child 1995;72:362-8

15 Brook CGD. Which children should receive growth hormone treatment? Reserve it for the GH deficienct. Arch Dis Child 2000;83:176.

\section{POSTCARD FROM AFRICA}

\section{Hospital management of asthma}

thought it would be easy: basic guidelines for hospital management of asthma. How wrong I was.

Constructing clinical guidelines is one of the tasks I've taken on for my year in Oshakati Hospital as a RCPCH/VSO paediatric fellow. Not wanting to be just another Oshilumbo who breezes in expecting the locals to do as I say, I set about trying to introduce my colleagues to evidence based medicine. Current journals and textbooks are hopelessly scarce but the Internet is accessible, and Namibia, classified by the World Bank as a lower middle income nation, qualifies for free online access to many journals.

So, I bravely said, don't just do what I say, demand evidence (thereby asking them to do as I say). That was the first catch-there is very little evidence for a whole host of conditions one sees here; I'm still trying to work out how best to treat Onyalai, (a bleeding disorder that claims a child every month or so). Furthermore, evidence that does exist often has little relevance: not giving antibiotics for a sore throat may make sense in the UK, but after you've seen three cases of rheumatic heart disease that morning and the family are about to travel 50 miles back to their village-believe me, you too would over treat with antibiotics.

But asthma, that's got to be easier; so much research, so many consensus statements, so many professionally designed guidelines.

The current in-house management for acute attacks relies on small doses of nebulised salbutamol with regular adrenaline. Metered dose inhalers are occasionally used for maintenance therapy when stocks of oral salbutamol run out but inhaled steroids are not used or even available (despite being on the Essential Drug List). Preventative therapy relies on theophylline and long courses of daily prednisolone.

I set out to encourage the use of standardised regular doses of nebulised salbutamol in preference to adrenaline for acute attacks. However, a Medline search I demonstrated to my colleagues to find randomised controlled trials revealed surprisingly few in favour of salbutamol, and fewer still for the dose I was recommending. It has been quite an experience to try to find trials to support practices that I regard as commonplace. Often the evidence is not there, how then can I advocate a change, or know that "my way" is indeed better?

Trials for outpatient maintenance therapy were a little more forthcoming. I could show inhaled salbutamol (and steroids) definitely work, and a few nice papers showing the effectiveness of homemade spacer devices. So having ascertained that salbutamol inhalers were in stock I considered it my duty to drink as much fizzy pop as was needed to keep the department in steady supply of plastic bottle spacers. It didn't take too long before I was stumped again; the inhalers were just cartridge refills without the plastic delivery casing. Just how does that help? Surely the cost saving is minimal, and now we have several hundred unusable inhaler refills. Not to be defeated I modified my plastic bottle so that the cartridge can be pressed directly into a small

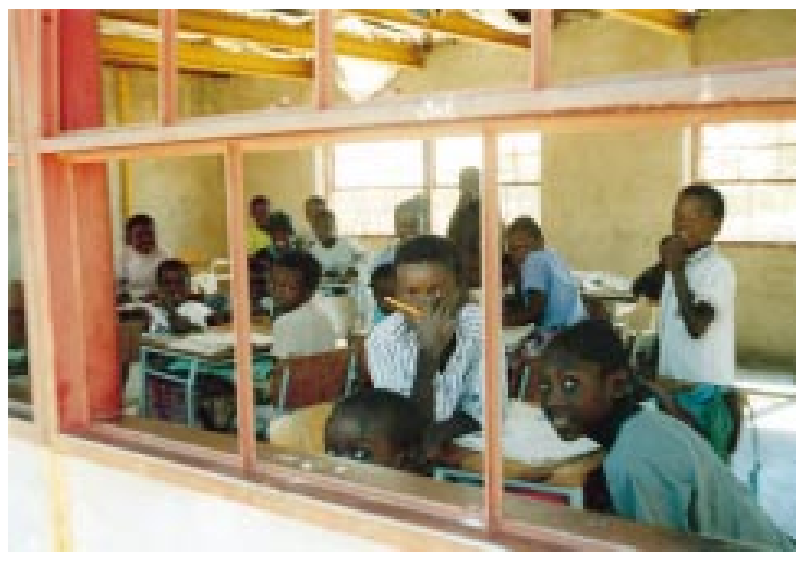

hole, delivering a pleasing mist of beta2 agonist which a few well trained children have dutifully inhaled "fudha, fudha!" (breathe, breathe!).

To date, my efforts have yielded little reward; most parents patiently watch, then puzzlingly ask if they can now have some proper medicine and leave without their plastic bottle. A paediatric nurse mother demonstrated to me that her child's inhaler worked much better when used in a "breath freshener" fashion than with the silly bottle thing.

So maybe there's a compromise to be reached, maintenance therapy for any chronic condition is notoriously difficult to manage and sustain anywhere, never mind in rural communities in Africa where, for many, fine tuned health is an unattainable luxury.

$I^{\prime} m$ starting to comprehend the questions that must be asked of every trial and intervention: Is this relevant to my target population? And is this acceptable by my target population?

I'm prescribing oral salbutamol occasionally, the patients are happier and the clinic sister is satisfied that my consultation times are approaching those of my colleagues ( 3 to 5 minutes maximum). Theophylline remains the mainstay of background control but at least prednisolone courses are shorter. I still battle on with my inhalers and spacers and they've even let me include them in the new guidelines. Maybe not as many children are leaving with spacers as I'd like, but at least now that I'm drinking less fizzy pop my teeth stand a chance.

R Tomlinson 